\title{
How are observed actions mapped to the observer's motor system? Influence of posture and perspective
}

\author{
Kaat Alaerts, Elke Heremans, Stephan P. Swinnen, Nicole Wenderoth* \\ Motor Control Laboratory, Research Center for Movement Control and Neuroplasticity, Department of Biomedical Kinesiology, \\ Group Biomedical Sciences, Katholieke Universtiteit Leuven, Tervuursevest 101, B-3001 Heverlee, Belgium
}

\section{A R T I C L E I N F O}

\section{Article history:}

Received 25 June 2008

Received in revised form 2 September 2008

Accepted 18 September 2008

Available online 24 September 2008

\section{Keywords:}

Mirror neurons

Action observation

Cortical excitability

Transcranial magnetic stimulation

Laterality

\begin{abstract}
A B S T R A C T
Previous studies using transcranial magnetic stimulation (TMS) have shown that during the observation of actions performed by others, the observer's primary motor cortex (M1) becomes facilitated in a highly muscle specific fashion. Here, we used TMS to explore the effect of posture, perspective and body side on muscle specific facilitation of left M1. Subjects viewed video's showing left and right hand extension (palm-down) movements from a first person or third person perspective with their hand posture either congruent (palm-down) or incongruent (palm-up) to the posture of the observed model.

Data indicated that facilitation of left M1 was substantially different for observing actions executed with the right (contralateral) or left (ipsilateral) hand. For right hand actions, facilitation of left M1 was shown to be highly specific to the muscle used in the observed action ('intrinsic mapping'). During the observation of left hand stimuli, only half of the subjects displayed this muscle specific facilitation, whereas in the other half, M1 was facilitated according to the observed movement direction ('extrinsic mapping'). Absolute effect magnitude was particularly high when right hand actions were observed from a first person perspective, whereas, for left hand actions, the third person perspective was more efficient. The degree of postural congruency between body parts of the observer and observed model only mildly influenced M1 facilitation. Since action observation is increasingly considered in rehabilitation therapies, the present findings may help identifying the most effective conditions for stimulating the motor system during action observation.
\end{abstract}

\section{Introduction}

In a socially dynamic world, basic understanding and imitation of others' gestures and movements is fundamental to human communicative behaviour and observational learning. A neural circuitry that is associated with these cognitive functions has been discovered in the macaque brain (Di Pellegrino, Fadiga, Fogassi, Gallese, \& Rizzolatti, 1992). The cells of this circuitry, the so-called 'mirror neurons' have the striking property not only to fire when the monkey performs an action, but also when the monkey observes a similar action performed by someone else (Gallese, Fadiga, Fogassi, \& Rizzolatti, 1996; Rizzolatti \& Craighero, 2004; Rizzolatti, Fadiga, Gallese, \& Fogassi, 1996a).

Abbreviations: ECR, extensor carpi radialis; EMG, electromyography; FCR, flexor carpi radialis; M1, primary motor cortex; MEP, motor-evoked potential; RMSE root-mean-square error; rMT, rest motor threshold; TMS, transcranial magnetic stimulation.

* Corresponding author. Tel.: +32 163291 57; fax: +32 16329197.

E-mail address: Nici.Wenderoth@faber.kuleuven.be (N. Wenderoth)
A similar fronto-parietal 'mirror neuron system' has also been identified in the human brain (Buccino et al., 2001; Iacoboni et al., 1999; Rizzolatti et al., 1996b). With single-pulse transcranial magnetic stimulation (TMS), it was also shown that the observer's motor system 'resonates' with the observed movements such that muscles involved in a certain action become facilitated by merely observing this action (Brighina, La Bua, Oliveri, Piazza, \& Fierro, 2000; Fadiga, Craighero, \& Olivier, 2005; Fadiga, Fogassi, Pavesi, \& Rizzolatti, 1995; Gangitano, Mottaghy, \& Pascual-Leone, 2001; Strafella \& Paus, 2000). Recent research has shown that this resonating activity might provide a window into how the observed movement is mapped onto the observer's body scheme (Lamm, Fischer, \& Decety, 2007). In daily life, the actions of others are often observed in different ways, which can roughly be characterized according to three distinct dimensions. First, the presence or absence of postural congruency between the observer's and actor's physical state might influence the effectiveness of movement observation. For example, observing another person typing at a keyboard might induce more resonating activity when the observer's hand is in pronation instead of supination. Second, peers can be perceived from different perspectives. Here, we will refer 
to an egocentric perspective (Ego) when the observed movement orientation corresponds to that of the observer (as if the action would be performed by the observer him/herself). In the allocentric perspective (Allo), the observed movement orientation is opposed to that of the observer (i.e., when an action is performed by another person facing the observer). Third, the observed action might exhibit congruency in terms of the observed body side (i.e., observing a right hand movement is anatomically congruent with the observer's right but not left hand).

Action observation is increasingly considered in rehabilitation contexts (Buccino, Solodkin, \& Small, 2006; Ertelt et al., 2007; Mulder, 2007; Pomeroy et al., 2005), such that a deeper understanding of the effects of body side, posture, and perspective is crucial for identifying the most effective conditions for stimulation of the motor system during action observation. Moreover, observational training programs in ergonomics and recreational contexts may also benefit from this knowledge.

Here, we aimed to explore the effects of these three factors, posture, perspective and body side, on muscular motor facilitation during action observation. For this purpose, TMS was used to measure the level of motor facilitation of the right flexor and extensor carpi radialis muscles (FCR/ECR) during the passive observation of several video clips showing simple extension movements of a model's wrist. In a previous study, it was already established that this action observation paradigm is suited to reveal a coherent picture of muscle specific facilitation of the observer's motor system, i.e., a stronger facilitation of the extensor muscle compared to the flexor as a result of extension movement observation (Alaerts, Swinnen, \& Wenderoth, in press). Overall, we expected the general muscle specific facilitation response to be preserved, but its magnitude to vary as a function of posture, body side and perspective.

The effect of posture was assessed by varying the observer's hand position from palm-down to palm-up, i.e., postures were congruent when extension movements were observed with palmdown posture, and incongruent for observation with palm-up posture. To assess the effect of the observer's perspective, video clips were observed from either an egocentric or allocentric perspective. To assess the effect of body side, both left and right hand extension movements were shown. Since cortico-spinal excitability was measured only in right ECR and FCR muscles, body sides were congruent for observing right hand and incongruent for observing left hand movements.

We hypothesized that muscle specific facilitation is reduced: (i) when the postures of model and observer are incongruent, (ii) when the actions are observed from an allocentric as compared to egocentric perspective, and (iii) when 'incongruent' left hand actions are observed (Aziz-Zadeh, Maeda, Zaidel, Mazziotta, \& Iacoboni, 2002). However, an alternative hypothesis is that left hand actions shown from an allocentric perspective are particularly beneficial in facilitating left M1, as suggested by a large body of behavioural work showing that mirror imitation is a more natural behaviour than anatomical imitation in many daily live tasks (Bekkering, Wohlschlager, \& Gattis, 2000; Gleissner, Meltzoff, \& Bekkering, 2000; Wapner \& Cirillo, 1968).

\section{Materials and methods}

\subsection{Subjects}

Participants were 4 male and 10 female university students (age range 20-28) without any known neuromuscular disorders. None were involved in neuroscience programs. All participants were right-handed, as assessed with the Edinburgh Handedness Questionnaire (Oldfield, 1971) and were naive about the purpose of the experiment. Written informed consent was obtained before the experiment and all subjects were screened for potential risk of adverse effects during TMS. The experimental procedure as well as the informed consent were approved by the local Ethics Committee for Biomedical Research at the Katholieke Universiteit Leuven in accordance to The Code of Ethics of the World Medical Association (Declaration of Helsinki) (Rickham, 1964).

\subsection{Electromyographic recording and TMS}

Motor-evoked potentials (MEPs) were recorded simultaneously from the flexor (FCR) and extensor (ECR) carpi radialis muscles of the right forearm. Electromyographic (EMG) recordings were performed with disposable $\mathrm{Ag}-\mathrm{AgCl}$ surface electrodes (Blue Sensor SP), placed over the middle portion of the muscle belly and aligned with the longitudinal axis of the muscles. Responses were sampled at $5000 \mathrm{~Hz}$ (CED Power 1401, Cambridge Electronic Design, UK) amplified, band-pass filtered $(30-1500 \mathrm{~Hz})$, and stored on a PC for off-line analysis. Pre-stimulus EMC recordings were used to assess the presence of unwanted background EMG activity in the $50 \mathrm{~ms}$ preceding the magnetic pulse.

Focal transcranial magnetic stimulation (TMS) was applied by means of a 70 mm figure of eight coil connected to a Magstim 200 stimulator (Magstim, Whitland Dyfed, UK). The coil was positioned over the left hemisphere, tangentially to the scalp with the handle pointing backward and laterally at $45^{\circ}$ away from the midsagittal line, such that the induced current flow was in a posterior-anterior direction, approximately perpendicular to the central sulcus. The optimal scalp position was defined as the position from which MEPs with maximal amplitude were recorded in the right FCR. The rest motor threshold (rMT) was defined as the lowest stimulus intensity required to evoke MEPs in the right FCR of at least $50 \mu \mathrm{V}$ of amplitude in 5 out of 10 consecutive stimuli (Rossini et al., 1994). For all experimental trials, the stimulation intensity was $130 \%$ of the subjects' rMT and ranged from 43 to $69 \%$ (mean $54 \%$ ) of the maximum stimulator output. Though the parameter setting procedures were prioritised for FCR, MEPs were simultaneously obtained for the ECR. ECR stimulation parameters were assumed to be satisfactorily similar, due to the overlapping representations of forearm flexors and extensors (Scheiber, 1990), and the lower threshold of the forearm extensor muscles. Signal Software (2.02 Version, Cambridge Electronic Design, UK) was used for TMS triggering and EMC recordings.

\subsection{Stimuli and procedure}

Two digital video clips showing extension movements of a left or right wrist were presented randomly to the subjects from the egocentric (i.e., hand oriented away from the observer) (Fig. 1A) and allocentric perspective (i.e., hand oriented towards the observer) (Fig. 1B). To explore possible effects of congruency between the hand posture of the observer and the observed model, participants were instructed to position their hand either with palm-down (congruent posture) or with palm-up (incongruent posture) while observing the extension movements. Vision of their own hands and forearms was never allowed. The participants were instructed to relax their forearm muscles while resting on a soft cushion directly underneath the video-screen. Muscle relaxation was monitored and whenever EMG activity became apparent during data collection, the trial was discarded and repeated.

Only the hand was seen in the video clips and the hand action was such that the wrist was rhythmically moved up (by approximately $30^{\circ}$ ) and down from the table surface with a frequency of $1.0 \mathrm{~Hz}$ for a total duration of $10 \mathrm{~s}$ (Fig. 1). Since experimental video conditions (8) were compared with each other, a low-level control condition was included as baseline, i.e., a video showing only a yellow background without any limb movement (BASELINE). Participants sat in front of a Dell 1707 monitor (resolution, $1152 \times 870$ pixels; refresh frequency, $60 \mathrm{~Hz}$ ) on which the AVIvideo-files (audio-video interleaved) were displayed with a frame rate of $25 \mathrm{~Hz}$ (or frames per seconds). Video presentation timing was controlled by Blaxton Video Capture software (South Yorkshire, UK). The computer screen was mounted directly above the subject's hand such that the observed movement could easily be incorporated into the body scheme of the observer. For each perspective (Ego and Allo) the two video clips were presented 16 times in random order for each subject: 8 times with the observer's hand in palm-down posture, 8 times in palm-up posture. The control video was presented 32 times ( 16 times in palm-down posture/16 times in palm-up posture). During the observation of each video clip, two single TMS pulses were delivered at pseudo-random movement cycles, such that both stimulations were applied when the hand in the video reached the extreme 'up' position. The 'up' position was chosen because excitability of the observer's ECR was reliably increased when observed from the egocentric perspective even though similar results were obtained for the extreme 'down' position (Alaerts et al., in press). In total, 192 MEPs were recorded for each subject. The experimental session lasted approximately $90 \mathrm{~min}$ with a pause every $30 \mathrm{~min}$. Participants were instructed to pay full attention to the videos, such that they could report the type of video after each trial. After the experiment, participants scored their subjective level of attention by means of a questionnaire. An average score of $4 \pm 0.7$ on a value scale ranging from 0 to 5 was reported.

\subsection{Data analysis}

From the EMG data, peak-to-peak amplitudes of the MEPs were determined. Normalized MEP amplitudes (normMEP) were calculated as the percentage of the BASELINE MEP amplitude for each muscle and posture. This procedure controlled 
(A) Egocentric Perspective

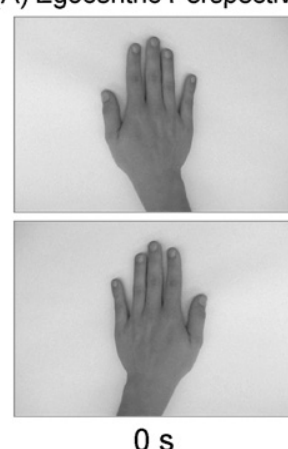

$0 \mathrm{~s}$

(B) Allocentric Perspective

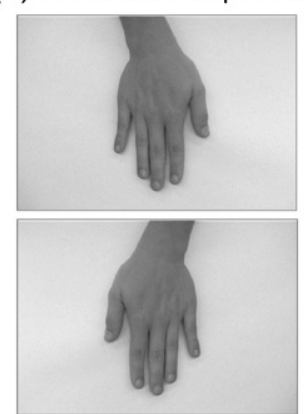

$0 \mathrm{~s}$

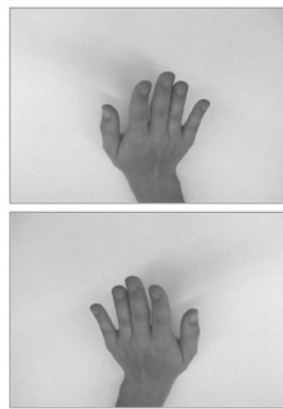

$1 \mathrm{~s}$

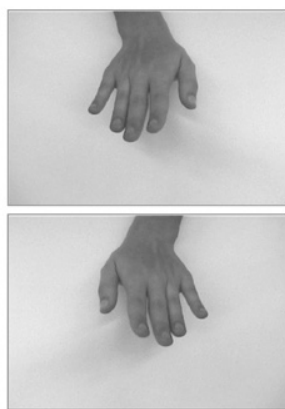

$1 \mathrm{~s}$

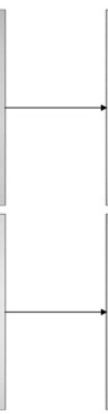

$\cdots$

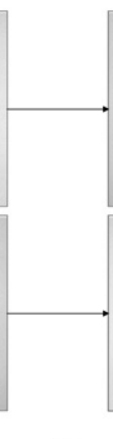

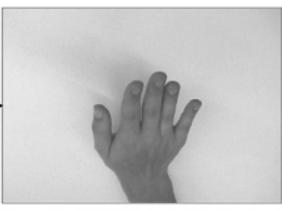

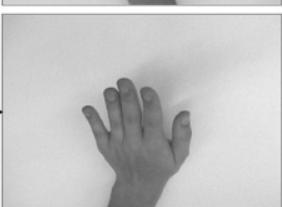

$9 \mathrm{~s}$

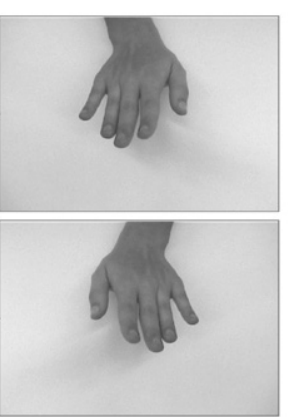

$9 \mathrm{~s}$
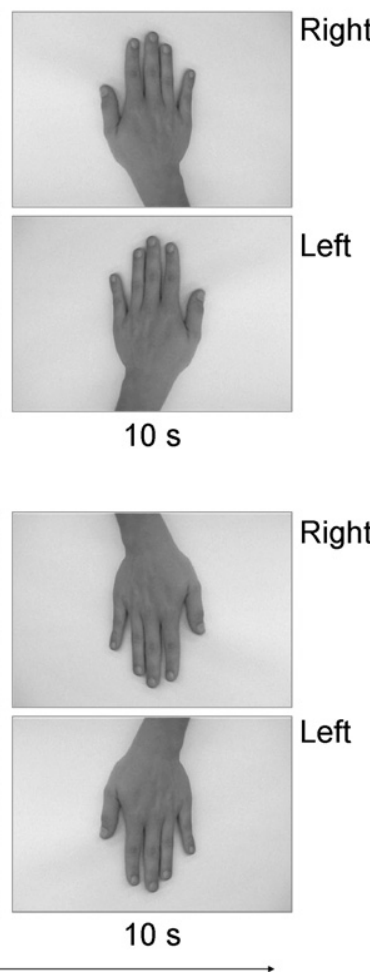

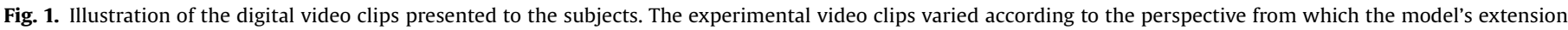
movement was presented (ego centric (A) versus allocentric (B) perspective) and the body side of the hand shown in the video clip (left versus right).

for potential confounds due to posture specific differences in the pre-innervation level of the recorded muscles and reduced inter-subject variability.

Background EMG was quantified by calculating the root-mean-square error (RMSE) across the $50 \mathrm{~ms}$ interval prior to TMS stimulation to ensure that subjects were completely relaxed during stimulation. Trials were removed from the analysis when EMG RMSE scores were larger than Q3+1.5(Q3 - Q1) with Q1, Q3 being the first and third quartile (computed separately for each observation condition and subject). One subject was excluded due to extremely high EMG activity in the FCR ( $124 \%$ of baseline RMSE). For the remaining subjects, $6 \%$ of all trials were discarded from further analyses. RMSE scores of the movement observation conditions were normalized relative to BASELINE scores.

\subsection{Statistics}

\subsubsection{Normalized MEP amplitudes}

To explore condition-induced modulations in normMEP for the ECR and FCR data were averaged for each muscle, observation condition (4 experimental and 1 baseline videos) and observer's posture (palm-up, palm-down) and subjected to a three-way analysis of variance (ANOVA) with repeated measurements. Withinsubjects factors were 'Posture' (palm-down, palm-up), 'Perspective' (Ego, Allo), and 'Body side' (Left, Right). To test whether the overall MEP response recorded from the ECR or FCR differed significantly from baseline, normMEPs from each muscle were averaged across observation conditions (for each subject) and a one-sample $t$-test was performed.

\subsection{2. $\triangle M E P$ scores}

Because we were primarily interested in the assessment of 'muscle-specific' changes in excitability during each observation condition, differences in response size were calculated between muscles ( $\triangle \mathrm{MEP}$ ) by subtracting the averaged normMEP values recorded from the FCR from those of the ECR (i.e., a positive $\triangle \mathrm{MEP}$ indicates higher ECR than FCR excitability). The calculation of $\triangle$ MEP scores is illustrated on the basis of exemplary data of a typical subject in Fig. 2. In addition to the normMEP results, $\triangle$ MEP scores can be interpreted as a measurement of 'muscle-specific responses' or 'muscular mapping' induced by the movement observation conditions. No difference between flexor and extensor facilitation $(\triangle M E P=0)$, would imply poor muscular mapping whereas a large positive value reflects a selective ECR response to the observed extension movement. Previous findings from our lab (Alaerts et al., in press) have shown that observing extension movements from an egocentric perspective leads to a highly muscle specific facilitation, i.e., positive $\triangle$ MEP scores (Fig. 2). With the present design, we expected muscle specific facilitation to be preserved for each observation condition (positive $\triangle \mathrm{MEP}$ scores), but its magnitude to vary as a function of posture, perspective and body side. $\triangle$ MEP values were calculated separately for each subject and each experimental observation condition. Statistics were calculated by means of planned comparisons using the non-parametric Wilcoxon Matched Pairs Test because data were not normally distributed. Bonferroni correction was applied when multiple, dependent comparisons were performed.

\subsubsection{RMSE scores}

To assess whether the MEP scores were confounded by modulations in background EMG, RMSE scores were expressed as the percentage difference from baseline. Data were averaged separately for each muscle, observation condition (4 experimental and 1 baseline videos) and observer's posture (palm-up, palm-down) and subjected to an ANOVA with repeated measures using the same factors as above. For all analyses, the level of statistical significance was set to $\alpha=0.05$.

\section{Results}

Normalized MEP amplitudes recorded from the ECR and FCR are reported in Table 1 for each observation condition. The ANOVA revealed a two-way interaction of 'Posture $\times$ Body side' for the normMEPs recorded from the FCR $[F(1,12)=9.01, p=0.01]$, which indicated that MEP responses of the FCR were higher for observing left hand actions with incongruent postures than with congruent postures. Overall, FCR excitability was low for observing right hands with either posture (Table 1 ). For the normMEPs recorded from the ECR muscle, a significant two-way interaction of 'Perspective $\times$ Body side' was found $[F(1,12)=6.81, p=0.02]$, which indicated that ECR facilitation was higher when right hand actions were observed from the egocentric perspective as compared to the allocentric perspective. On the other hand, the observational perspective did not influence ECR facilitation when left hands were observed (Table 1 ).

An additional one-sample t-test was applied after normMEPs were averaged across conditions. It revealed that, overall, 


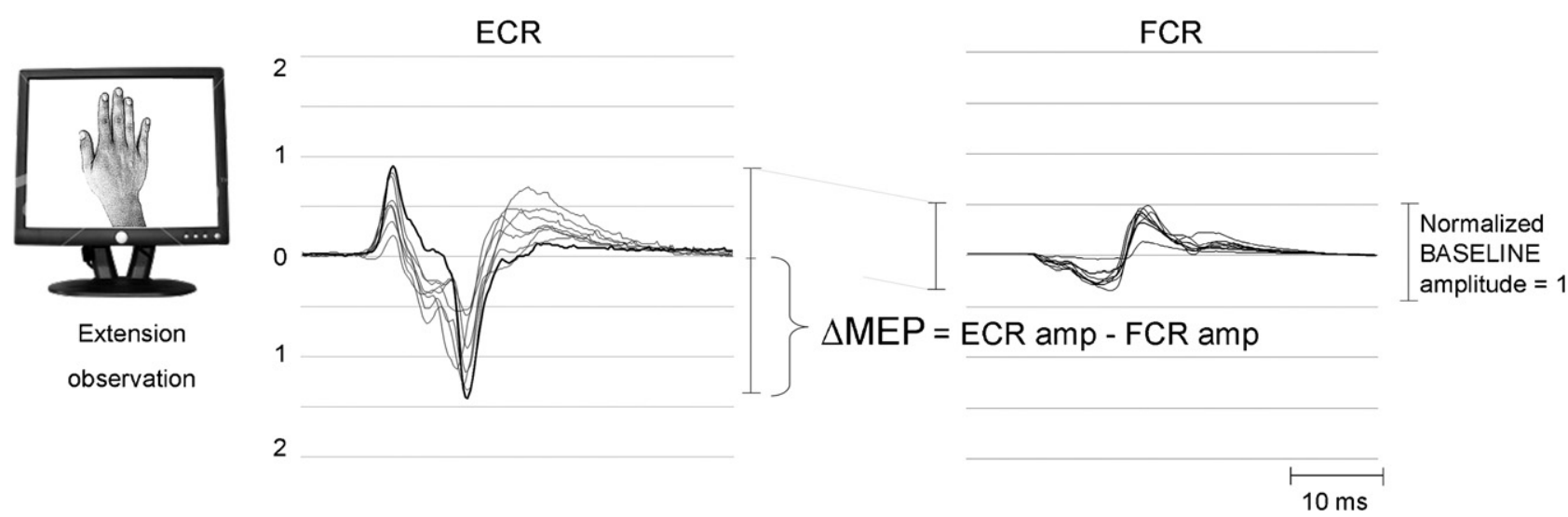

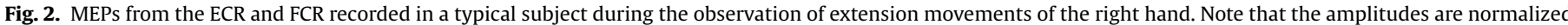

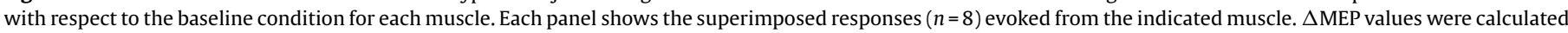

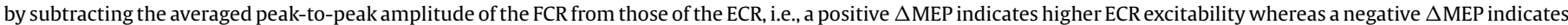
higher FCR excitability.

ECR excitability was significantly increased compared to baseline $[t=2.6, p=0.02]$, whereas no significant over-all change was observed for FCR excitability [ $t=0.5, p=0.62]$.

Based on the significant interactions revealed by the original normMEP responses, we tested analogue interactions for the $\triangle M E P$ scores using planned comparisons. $\triangle \mathrm{MEP}$ represents the difference in corticospinal facilitation of flexor and extensor of the observer and is considered to reflect the effectiveness of muscle specific mapping of the observed movement onto the motor system. Because the results can best be understood in light of all three factors, Fig. 3 shows $\triangle$ MEP scores for all observation conditions.

\subsection{Interaction between body side and posture}

The data in Fig. $3 \mathrm{~A}$ and $\mathrm{C}$ show that the $\triangle \mathrm{MEP}$ scores differed substantially when left as compared to right hand actions were observed. More specifically, $\triangle \mathrm{MEP}$ scores were substantially larger for congruent than for incongruent postures when the video showed left hand movements (Fig. 3A), whereas no difference between postures was observed when right hand actions were displayed in the video (Fig. 3C). This observation was confirmed by a planned comparison for the 'Body side $\times$ Posture' interaction on the $\triangle$ MEP data $[Z=2.13, p=0.03]$.

For left hand observation with incongruent postures, $\triangle \mathrm{MEP}$ scores were shown to be close to 0 (Fig. 3A). This may reflect two different mechanisms: First, it is possible that observing left hand extension did not selectively increase ECR excitability over

\section{Table 1}

Normalized peak-to-peak MEP amplitude scores recorded during the observation of video clips showing extension movements of a left or right hand [expressed as mean changes from baseline MEP size (in \%) \pm standard errors]. Clips were observed from an ego- or allo-centric perspective, with the observer's hand positioned with congruent or incongruent postures. Data for each condition were subjected to onesample $t$-tests with Bonferroni correction to determine whether excitability differed significantly from baseline. Significance $(p<0.05)$ is indicated by *

\begin{tabular}{|c|c|c|c|c|}
\hline Body side & Perspective & Posture & ECR & FCR \\
\hline \multirow{4}{*}{ Left } & \multirow{2}{*}{ Egocentric } & Congruent & $11.26 \pm 6.11$ & $-4.27 \pm 3.65$ \\
\hline & & Incongruent & $4.51 \pm 4.20$ & $8.41 \pm 5.87$ \\
\hline & \multirow{2}{*}{ Allocentric } & Congruent & $13.37 \pm 7.23$ & $0.01 \pm 5.04$ \\
\hline & & Incongruent & $6.63 \pm 6.11$ & $12.20 \pm 5.81$ \\
\hline \multirow{4}{*}{ Right } & \multirow{2}{*}{ Egocentric } & Congruent & $19.17 \pm 4.51^{*}$ & $0.17 \pm 5.32$ \\
\hline & & Incongruent & $8.84 \pm 6.40$ & $-9.79 \pm 2.64^{*}$ \\
\hline & \multirow[b]{2}{*}{ Allocentric } & Congruent & $7.29 \pm 3.90$ & $4.84 \pm 4.00$ \\
\hline & & Incongruent & $1.36 \pm 4.37$ & $0.26 \pm 4.65$ \\
\hline
\end{tabular}

and above FCR excitability indicating poor resonating activity of the observer's M1. Second, it might be that the mapping of the observed movement onto the observer's motor system differed across subjects such that some exhibited a muscle specific facilitation (i.e., selective ECR facilitation) whereas others exhibited the converse facilitation pattern (i.e., FCR facilitation). To analyse the behaviour of the individual subjects, the distribution of $\triangle \mathrm{MEP}$ scores recorded during the observation of left hands with incongruent postures was determined (Fig. 3B). The distribution was bimodal and deviated significantly from normality [Shapiro Wilk W test, $p=0.01$ ] as most subjects exhibited large $\triangle$ MEP values which were either negative or positive. Thus, for approximately half of the subjects, ECR excitability was larger than FCR excitability, indicating a muscle specific facilitation pattern. By contrast, in the remaining half, excitability was clearly higher in the FCR than the ECR, even when the model in the video activated the extensor muscle. This finding suggests that the latter subjects obeyed a different mapping between the observed movement and their own motor system which resonated with respect to the observed movement direction of the hand ('extrinsic mapping'), rather than to the observed muscle activation ('intrinsic mapping'). By analogy, the 'Posture $\times$ Body side' interaction on the normMEP data recorded from the FCR already indicated that FCR excitability was maximal for observing left hand motion with incongruent postures (Table 1)

We further explored this unexpected result by performing a planned comparison between congruent and incongruent postures on the absolute $\triangle \mathrm{MEP}$ values recorded during the left hand observation conditions. Note that this analysis is not sensitive to between-subjects differences in the observation-to-observer mapping strategy (i.e., intrinsic or extrinsic), but tests whether the response magnitude was similar for congruent or incongruent postures. We found that the absolute $\triangle$ MEP responses were only slightly smaller for incongruent $(23 \pm 4 \%)$ as compared to congruent postures $(25 \pm 4 \%)$ such that no significant posture effect was found [planned comparisons, $Z=0.24, p=0.8$ ].

Taken together, the initial comparison between congruent versus incongruent postures unmasked that subjects mapped the observed movements differently to their own body scheme (intrinsic versus extrinsic coordinate frame) (Fig. 3B), but only when left hand actions were observed, not right hand actions. However, when the extrinsic versus intrinsic nature of the mapping was disregarded, the absolute response magnitude was similar across postures and substantially larger than baseline. This unexpected finding will be discussed further in Section 4 . 


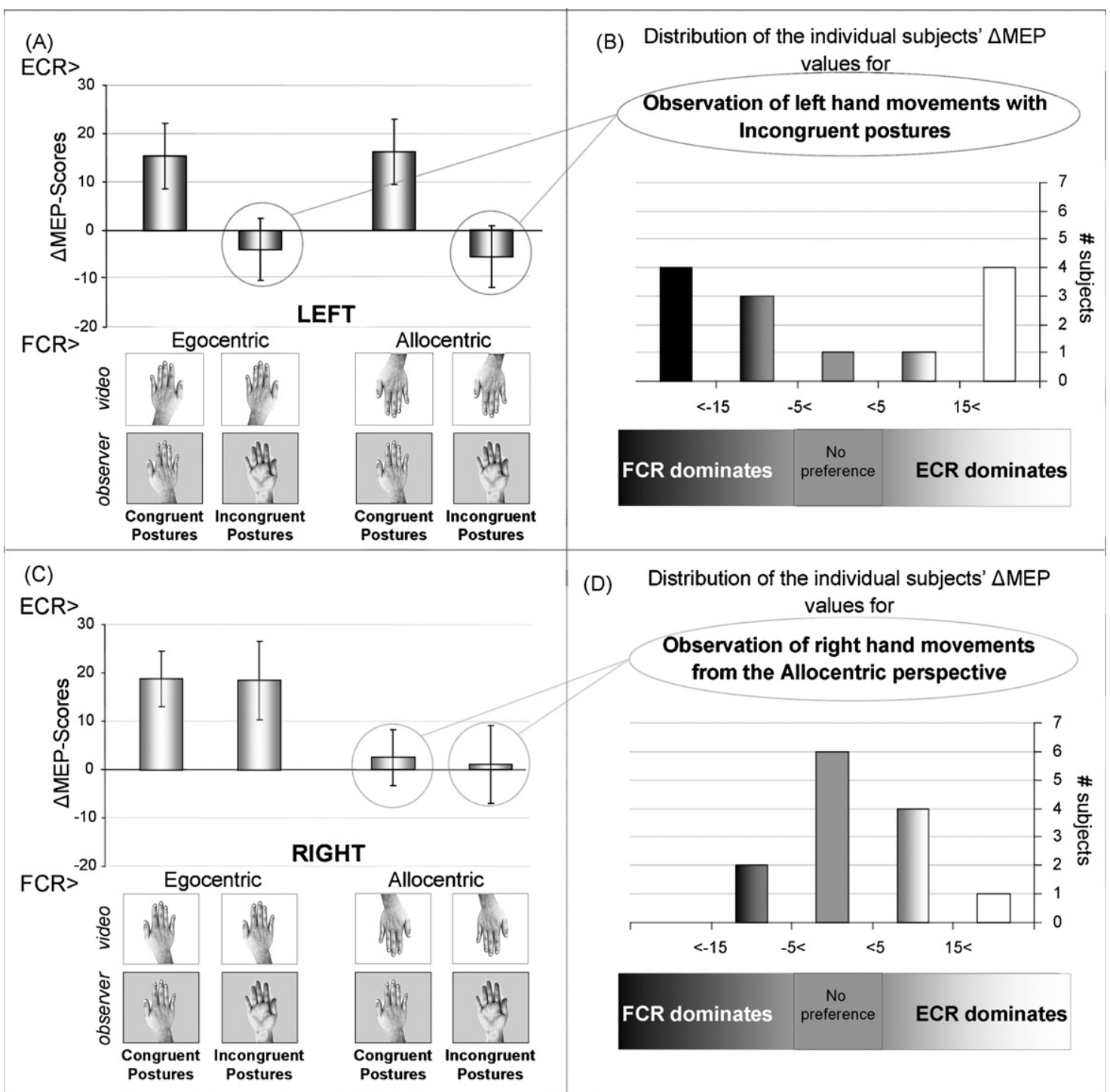

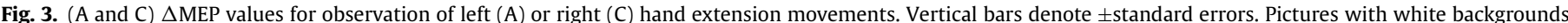

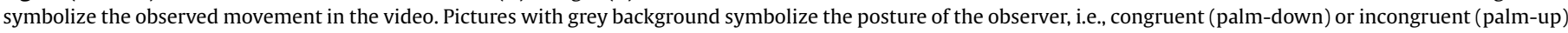

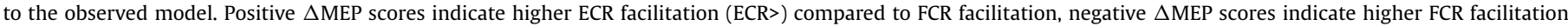

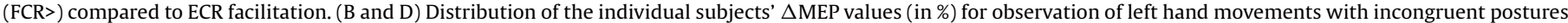

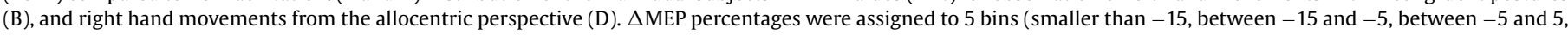
between 5 and 15, larger than 15).

\subsection{Interaction between body side and perspective}

For right hand movements, $\triangle \mathrm{MEP}$ scores were substantially higher for observing videos from the egocentric, compared to the allocentric perspective (Fig. 3C). By contrast, the factor 'perspective' did not modulate $\triangle$ MEP scores when left hand actions were observed (Fig. 3A). This observation was only partly supported by the statistics revealing a trend towards significance $[Z=1.71$ $p=0.08]$ when the 'Body side $\times$ Perspective' interaction was tested with planned comparisons. $\triangle \mathrm{MEP}$ scores close to 0 indicate either that there was no differential facilitation for ECR and FCR or that subjects mapped the observed movement differently onto their own motor system (i.e., intrinsic or extrinsic). The distribution of the individual subjects' $\triangle \mathrm{MEP}$ values (Fig. 3D) shows that the majority of the subjects exhibited $\triangle$ MEP scores close to 0 , indicating no difference in facilitation of either the ECR or FCR (Fig. 3D). Inspecting Table 1, it can be seen that corticospinal excitability of the ECR and FCR was relatively close to baseline when subjects observed right hand movements from an allocentric perspective and, particularly, when they were additionally positioned in incongruent postures. Thus, observing right hand movements from an allocentric perspective did not substantially increase corticospinal excitability of neither the ECR nor the FCR (Table 1).

Considering that watching left hand actions resulted in differential observation-to-observer mappings across subjects, we performed a planned comparison for 'Body side $\times$ Perspective' effects on the absolute $\triangle$ MEP scores recorded during left and right hand observation (Fig. 4). This revealed a significant interaction effect $[Z=2.97, p<0.003]$ such that, for observing left hand movements, response magnitude was higher for the allocentric ( $29 \pm 2 \%)$ than for the egocentric perspective ( $20 \pm 3 \%)$, whereas for observing right hand movements, the opposite effect was found (egocentric perspective $24 \pm 4 \%$ > allocentric perspective $15 \pm 2 \%$ ) (Fig. 4 ).

\subsection{Background EMG data}

Background EMG was generally small and condition specific modulations were minimal. This was tested by conducting similar 


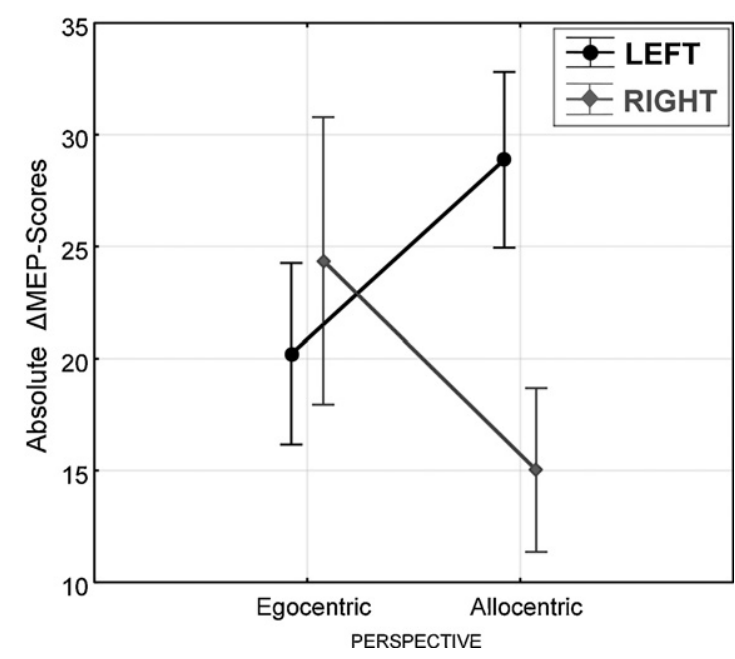

Fig. 4. Absolute $\triangle \mathrm{MEP}$ scores for observation of left and right hand extension movements. Vertical bars denote \pm standard errors. The significant 'Perspective $\times$ Body side' interaction indicated that left hand actions evoke larger absolute responses when observed from an allocentric perspective, whereas for right hand actions, absolute responses were maximal when observed from an egocentric perspective.

ANOVA's (within factors, 'Posture', 'Perspective' and 'Body side') to the corresponding background EMG data (normalized RMSE-scores). None of the above Posture $\times$ Body side or Perspective $\times$ Body Side interaction effects were found significant [all $F<2.5, p>0.15$ ], which indicated that the MEP amplitude scores were not confounded by modulations in background EMG.

\section{Discussion}

The influence of posture, perspective, and body side on movement observation was investigated by means of a full factorial design. The analyses revealed substantial differences in the responses of the observers' left M1 when right (contralateral) versus left (ipsilateral) hand actions were observed.

(1) When observing right hand actions, all subjects mapped the observed movements in a highly muscle specific way to their own body (intrinsic mapping). By contrast, when left hand actions were observed, the observation-to-observer mapping differed across subjects, such that approximately half of the subjects appeared to exhibit a muscle specific (intrinsic) and the other half a direction specific (extrinsic) mapping.

(2) When these differences in observation-to-observer mapping were disregarded and the absolute response magnitude was compared, we found that right hand actions induced the strongest effects when observed from an egocentric perspective, whereas left hand movements yielded the strongest responses when observed from an allocentric perspective.

\subsection{Different observation-to-observer mappings for left but not for right hand actions}

We examined whether the degree of postural congruency between the observer's and model's hand position influenced the effectiveness of muscular motor mapping as indicated by changes of corticospinal excitability in left M1. To do so, we varied the posture of the observer from palm-down to palm-up position, while viewing extension movements (model's hand in palm-down) of a model's left and right hand. When right hand extension movements were observed, highly muscle specific facilitation of the extensor over and above the flexor was found, irrespective of whether the observer's posture was congruent or incongruent. These results are in excellent agreement with previous studies which also found only minor influences of posture on the process of muscle specific mapping of observed movements (Urgesi, Moro, Candidi, \& Aglioti, 2006) (Alaerts et al., in press).

To our surprise, we found a different behaviour when left hand movements were observed. More specifically, our posture manipulations unmasked that approximately half of the subjects displayed the expected muscle congruent facilitation pattern (i.e., maximal extensor facilitation during extension movements observation), whereas the other half exhibited the opposite facilitation pattern, i.e., when postures were incongruent, flexor excitability was larger than extensor excitability even though an extension movement was shown in the video. The latter subjects possibly 'resonated' with the observed movement according to direction in extrinsic space, i.e., when they observed an upward movement; excitability was highest in their own upward moving muscle (i.e., the flexor muscle since hands were positioned in palm-up posture). It is important to note that direction specific (extrinsic) versus muscle specific (intrinsic) mapping can only be disentangled with incongruent posture conditions. The reason is that for congruent postures, direction and muscle parameters co-vary, such that both intrinsic and extrinsic mapping result in extensor facilitation.

The directional mapping findings should be interpreted with caution because it was based on a limited number of observation conditions (i.e., conditions in which left hands were observed with incongruent postures), and it was only found in approximately half of the subjects. Nevertheless, it is tempting to speculate on the putative mechanism underlying this finding. Single cell recordings in monkeys have shown that neurons of the inferior frontal gyrus (IFG) encode movements mainly with respect to direction in extrinsic space (Kakei, Hoffman, \& Strick, 2001) whereas M1 contains additional neurons encoding movements in intrinsic space, i.e., with respect to muscles and joints (Kakei, Hoffman, \& Strick, 1999; Kakei, Hoffman, \& Strick, 2003). We speculate that in our study, the interaction between IFG and M1 may have been different when left (ipsilateral) as compared to right (contralateral) hand actions were observed, such that some subjects expressed an extrinsic mapping when the left (ipsilateral) hand was observed. This is in general agreement with a functional imaging study of Aziz-Zadeh, Koski, Zaidel, Mazziotta, and Iacoboni (2006) showing that IFG tended to be more activated when ipsilateral as opposed to contralateral finger movements were observed (Aziz-Zadeh et al., 2006). It remains puzzling why direction specific facilitation of M1 was only measured in some subjects. One potential explanation is that the intrinsic versus extrinsic mapping might depend on the subjects' degree of handedness. However, this was not confirmed by our data. Alternatively, the subjects' movement repertoire and experience can influence observation-to-observer mappings during action observation (Calvo-Merino, Glaser, Grezes, Passingham, \& Haggard, 2005; Maeda, Chang, Mazziotta, \& Iacoboni, 2001). It is possible that some of our participants were more experienced in directional mapping of observed and executed movements, as often required during dance or aerobics. By any means, the aforementioned interpretations are speculative and further research is warranted to firmly establish the existence of differential observation-to-observer mappings as well as their underlying mechanisms.

\subsection{Larger absolute responses are evoked when right hand actions are observed from an egocentric perspective and left hand actions from an allocentric perspective}

Realizing that subjects appeared to exhibit different observation-to-observer mappings, we disregarded these inter- 
individual differences and compared the absolute response magnitude across conditions. For right hand actions, response magnitudes of M1 were high when the movies were observed from an egocentric perspective, whereas corticospinal excitability did not change substantially from baseline when observing the movies from an allocentric perspective (Fig. $3 \mathrm{C}$ and 4 ). This result is in agreement with previous findings of Maeda, Kleiner-Fisman, and Pascual-Leone (2002).

Conversely, when left hand actions were observed, the response magnitude of M1 facilitation was larger for the allocentric than the egocentric perspective (Fig. 4). Why does left M1 respond more strongly to left (ipsilateral) than to right (contralateral) hand actions when shown from an allocentric perspective? This finding can best be interpreted in the light of previous 'allocentric' imitation studies, showing a strong preference towards 'mirror' or so-called 'specular' imitation' of others' actions (i.e., model moves the left hand, imitator moves the right hand) as compared to 'anatomic' imitation (i.e., model and imitator move both their left hand) when the actors face each other during imitation (Bekkering et al., 2000; Gleissner et al., 2000; Schofield, 1976; Wapner \& Cirillo, 1968). Imaging studies have also emphasized this 'mirror' imitation preference by showing that typical 'mirror areas' (e.g., bilateral inferior frontal and right posterior parietal cortex) are more strongly activated during mirror than anatomic imitation (Koski, Iacoboni, Dubeau, Woods, \& Mazziotta, 2003). To our best knowledge, our study revealed the first TMS results supporting this hypothesis. Indeed, when viewed from the allocentric perspective, left hand actions induce a 'mirror-like' configuration ensuring that the general shape or 'gestalt' of the hand is congruent between the model and observer (Fig. 3A, 3rd bar). As such, our findings extend these previous results by showing that the strong tendency toward 'mirror-like' or spectral imitation as opposed to anatomical imitation, is also reflected in the excitability changes measured at the level of the primary motor cortex during the mere observation of actions. Accordingly, for observation from the allocentric perspective, left (ipsilateral) movement stimuli appeared to be mapped more effectively onto the observer's motor system than right (contralateral) movements.

\subsection{Movement observation in rehabilitation}

Action observation is increasingly considered in the context of rehabilitation and motor learning (Buccino et al., 2006; Ertelt et al., 2007; Mulder, 2007; Pomeroy et al., 2005). To optimize the recruitment of cortical motor areas in the lesional hemisphere during action observation, our data suggest the utilization of movement stimuli showing the contralateral limb when presented from an egocentric perspective (first person), and the ipsilateral limb when presented from an allocentric perspective (third person). Intuitively, the incorporation of an observed motion will likely benefit from a high degree of congruency between the posture of the observer and that of the observed model. However, based on the present results, no substantial differences were found in overall motor activation due to postural variations.

The application of movement observation is theoretically based on the finding that cortical motor circuits are activated in a similar way during movement observation as during execution (Fadiga et al., 2005; Fadiga et al., 1995; Strafella \& Paus, 2000). Conversely, increased inhibition is measured at the spinal level, presumably to prevent the overt replication of the seen action (Baldissera, Cavallari, Craighero, \& Fadiga, 2001). Although movement execution is known to depend on the coordinated interplay of both excitatory and inhibitory circuits at the cortical level, it is not clear whether movement observation also evokes selective cortical inhibition in a similar way as needed during movement execution. This is an important question for further research and, particularly, in the context of rehabilitation.

\section{Summary and conclusions}

The present study suggests that M1 of the left hemisphere responds to observing actions executed with the right (contralateral) or left (ipsilateral) hand. For right limb stimuli, activations in left $\mathrm{M} 1$ are highly specific to the muscle used in the observed action ('intrinsic mapping'). During the observation of left limb stimuli, only part of the subjects display this muscle specific activation of left M1, whereas in others, primary motor cortex appears to resonate with respect to the observed movement direction of the hand ('extrinsic mapping'). Irrespective of mapping strategy, right limb stimuli evoke the highest responses when observed from an egocentric perspective (first person), whereas responses to left stimuli are maximized when observed from the allocentric perspective (third person). Overall, the degree of postural congruency between observer/model body parts only mildly influences M1 responses.

\section{Acknowledgements}

Support for this study was provided through grants from the Flanders Fund for Scientific Research (Projects G.0292.05 and G.0577.06). This work was also supported by Grant P6/29 from the Interuniversity Attraction Poles program of the Belgian federal government.

\section{References}

Alaerts, K., Swinnen, S.P., \& Wenderoth, N., Is the human primary motor cortex activated by muscular or direction-dependent features of observed movements? Cortex, in press.

Aziz-Zadeh, L., Maeda, F., Zaidel, E., Mazziotta, J., \& Iacoboni, M. (2002). Lateralization in motor facilitation during action observation: A TMS study. Experimental Brain Research, 144, 127-131.

Aziz-Zadeh, L., Koski, L., Zaidel, E., Mazziotta, J., \& Iacoboni, M. (2006). Lateralization of the human mirror neuron system. Journal of Neuroscience, 26, 2964-2970.

Baldissera, F., Cavallari, P., Craighero, L., \& Fadiga, L. (2001). Modulation of spinal excitability during observation of hand actions in humans. European Journal of Neuroscience, 13, 190-194.

Bekkering, H., Wohlschlager, A., \& Gattis, M. (2000). Imitation of gestures in children is goal-directed. Quarterly Journal of Experimental Psychology Section A: Human Experimental Psychology, 53, 153-164.

Brighina, F., La Bua, V., Oliveri, M., Piazza, A., \& Fierro, B. (2000). Magnetic stimulation study during observation of motor tasks. Journal of the Neurological Sciences, 174, 122-126.

Buccino, G., Binkofski, F., Fink, G. R., Fadiga, L., Fogassi, L., Gallese, V., et al. (2001). Action observation activates premotor and parietal areas in a somatotopic manner: An fMRI study. European Journal of Neuroscience, 13, 400-404.

Buccino, G., Solodkin, A., \& Small, S. L. (2006). Functions of the mirror neuron system: Implications for neurorehabilitation. Cognitive and Behavioral Neurology, $19,55-63$.

Calvo-Merino, B., Glaser, D. E., Grezes, J., Passingham, R. E., \& Haggard, P. (2005). Action observation and acquired motor skills: An fMRI study with expert dancers. Cerebral Cortex, 15, 1243-1249.

Di Pellegrino, G., Fadiga, L., Fogassi, L., Gallese, V., \& Rizzolatti, G. (1992). Understanding motor events: A neurophysiological study. Experimental Brain Research, 91, $176-180$.

Ertelt, D., Small, S., Solodkin, A., Dettmers, C., McNamara, A., Binkofski, F., et al. (2007). Action observation has a positive impact on rehabilitation of motor deficits after stroke. Neuroimage, 36(Suppl 2), T164-T173.

Fadiga, L., Fogassi, L., Pavesi, G., \& Rizzolatti, G. (1995). Motor facilitation during action observation: A magnetic stimulation study. Journal of Neurophysiology, 73, 2608-2611.

Fadiga, L., Craighero, L., \& Olivier, E. (2005). Human motor cortex excitability during the perception of others' action. Current Opinion in Neurobiology, 15, 213-218.

Gallese, V., Fadiga, L., Fogassi, L., \& Rizzolatti, G. (1996). Action recognition in the premotor cortex. Brain, 119, 593-609.

Gangitano, M., Mottaghy, F. M., \& Pascual-Leone, A. (2001). Phase-specific modulation of cortical motor output during movement observation. Neuroreport, 12 , 1489-1492.

Gleissner, B., Meltzoff, A. N., \& Bekkering, H. (2000). Children's coding of human action: Cognitive factors influencing imitation in 3-year-olds. Developmental Science, 3, 405-414. 
Iacoboni, M., Woods, R. P., Brass, M., Bekkering, H., Mazziotta, J. C., \& Rizzolatti, G. (1999). Cortical mechanisms of human imitation. Science, 286, 2526 2528.

Kakei, S., Hoffman, D. S., \& Strick, P. L. (1999). Muscle and movement representations in the primary motor cortex. Science, 285, 2136-2139.

Kakei, S., Hoffman, D. S., \& Strick, P. L. (2001). Direction of action is represented in the ventral premotor cortex. Nature Neuroscience, 4, 1020-1025.

Kakei, S., Hoffman, D. S., \& Strick, P. L. (2003). Sensorimotor transformations in cortical motor areas. Neuroscience Research, 46, 1-10.

Koski, L., Iacoboni, M., Dubeau, M. C., Woods, R. P., \& Mazziotta, J. C. (2003). Modulation of cortical activity during different imitative behaviors. Journal of Neurophysiology, 89, 460-471.

Lamm, C., Fischer, M. H., \& Decety, J. (2007). Predicting the actions of others taps into one's own somatosensory representations-a functional MRI study. Neuropsychologia, 45, 2480-2491.

Maeda, F., Chang, V. Y., Mazziotta, J., \& Iacoboni, M. (2001). Experience-dependent modulation of motor corticospinal excitability during action observation. Experimental Brain Research, 140, 241-244.

Maeda, F., Kleiner-Fisman, G., \& Pascual-Leone, A. (2002). Motor facilitation while observing hand actions: specificity of the effect and role of observer's orientation. Journal of Neurophysiology, 87, 1329-1335.

Mulder, T. (2007). Motor imagery and action observation: Cognitive tools for rehabilitation. Journal of Neural Transmission, 114, 1265-1278.

Oldfield, R. C. (1971). The assessment and analysis of handedness: The Edinburgh inventory. Neuropsychologia, 9, 97-113.

Pomeroy, V. M., Clark, C. A., Miller, J. S. G., Baron, J. C., Markus, H. S., \& Tallis, R. C. (2005). The potential for utilizing the "mirror neurone system" to enhance recovery of the severely affected upper limb early after stroke: A review and hypothesis. Neurorehabilitation and Neural Repair, 19, 4-13.

Rickham, P. P. (1964). Human experimentation. Code of ethics of the world medical association. Declaration of Helsinki. British Medical Journal, 2, 177.

Rizzolatti, G., \& Craighero, L. (2004). The mirror-neuron system. Annual Review of Neuroscience, 27, 169-192.

Rizzolatti, G., Fadiga, L., Gallese, V., \& Fogassi, L. (1996). Premotor cortex and the recognition of motor actions. Cognitive Brain Research, 3, 131-141.

Rizzolatti, G., Fadiga, L., Matelli, M., Bettinardi, V., Paulesu, E., Perani, D., et al. (1996). Localization of grasp representations in humans by PET 1. Observation versus execution. Experimental Brain Research, 111, 246-252.

Rossini, P. M., Barker, A. T., Berardelli, A., Caramia, M. D., Caruso, G., Cracco, R. Q., et al. (1994). Noninvasive electrical and magnetic stimulation of the brain spinal-cord and roots-basic principles and procedures for routine clinicalapplication-report of an IFCN committee. Electroencephalography and Clinical Neurophysiology, 91, 79-92.

Scheiber, MH. (1990). How might the motor cortex individuate movements. Trends in Neurosciences, 13, 440-445.

Schofield, W. N. (1976). Do children find movements which cross body midline difficult. Quarterly Journal of Experimental Psychology, 28, 571-582.

Strafella, A. P., \& Paus, T. (2000). Modulation of cortical excitability during action observation: A transcranial magnetic stimulation study. Neuroreport, 11, 2289-2292.

Urgesi, C., Moro, V., Candidi, M., \& Aglioti, S. M. (2006). Mapping implied body actions in the human motor system. Journal of Neuroscience, 26, 7942-7949.

Wapner, S., \& Cirillo, L. (1968). Imitation of a model's hand movements: Age changes in transposition of left-right relations. Child Development, 39, 887-894. 